\title{
L'aggettivo valanghivo riferito a valanga
}

\author{
Matilde Paoli
}

PUBBLICATO: 05 MARZO 2010

\section{Quesito:}

Maria Poloniato, insegnante di scuola secondaria superiore di San Donà di Piave, ci scrive di aver sentito, in un'edizione locale del $\mathrm{Tg} 3$, il sintagma attivitá valanghiva. Visto che l'aggettivo è registrato soltanto in ZINGARELLI, si chiede se il suo uso sia sia legittimo o, in caso contrario, quale potrebbe essere una formazione aggettivale derivata da valanga.

\section{L'aggettivo valanghivo riferito a valanga}

$\mathrm{E}$ ffettivamente l'aggettivo valanghivo è registrato solo da Zingarelli a partire dall'edizione 2003 come annunciato anche in un articolo di "Repubblica" del ro luglio 2002: "Sono più di duecento le parole che entreranno nell'edizione 2003 dello Zingarelli [...], vocaboli che hanno fatto una lunga anticamera, da valanghivo, parola nata nel 1992 per indicare una zona soggetta a valanghe, a omocodia..." (Ciboide, bancheggiare, valanghivo ecco le nuove parole del futuro). Non è da escludersi che, per la datazione, i redattori del dizionario si siano basati sulla prima delle tre sole attestazioni reperibili nel "Corriere della sera", risalente appunto al I992: «"Due potenziali neosuperfici valanghive" spiega con linguaggio tecnico il dottor Giovanni Peretti, direttore del centro nivometeorologico regionale di Bormio» e più sotto (sono sempre parole del dottor Peretti) "la nuova e importante area valanghiva alpina" (Michele Pusterla, L'incubo valanghe in Valtellina, 2 gennaio I992).

Sulla stampa l'uso si mostra assai raro (sei occorrenze su "Repubblica", non più di una per anno) e legato soprattutto al sintagma rischio valanghivo; in ambienti specialistici invece la voce è impiegata e risulta attestata già in precedenza: la si trova per esempio in L'Italia forestale e montana (vol. 34/35), pubblicato dall'Accademia Italiana di Scienze Forestali nel 1979, negli Atti del convegno annuale dell'Associazione geofisica italiana, risalenti al 1969, in Bibliografia Geofisica Italiana, a cura della stessa Associazione, del 1957, in Geofisica e meteorologia, edito dalla Società italiana di geofisica e meteorologia nel 1953, e ancora (l'attestazione piu antica che abbiamo rintracciato) nel Bollettino della Societá geologica italiana risalente all'anno I868. Anche le attestazioni più recenti appaiono legate ad ambienti specifici. Naturalmente è usato dalla comunità scientifica (è reperibile, per fare qualche esempio, nel Manuale di geomorfologia applicata di Mario Panizza, 2005, negli atti del convegno internazionale La difesa della montagna, tenutosi a Roma nel dicembre 2002, pubblicati nel 2003 a cura dell'Accademia nazionale dei Lincei, negli Studi trentini di scienze naturali: Acta geologica, editi dal Museo tridentino di scienze naturali ancora nel 2003). Valanghivo risulta inoltre impiegato da amministrazioni locali, ovviamente dell'area alpina (appare nei siti ufficiali della Provincia Autonoma di Bolzano, delle Regioni Autonome Valle d'Aosta e Friuli Venezia Giulia) e specialmente dalle loro Agenzie Regionali per la Protezione dell'Ambiente (si trova nei siti di ARPA di Piemonte e Veneto); lo usano poi i Servizi meteorologici di area alpina, le associazioni che hanno che fare con la montagna e con la gestione dei soccorsi (Club Alpino Italiano, Soccorso Alpino, Protezione Civile) e anche l'EIM, Ente Italiano della Montagna, direttamente connesso al Governo della Repubblica.

Le contestualizzazioni più frequenti, stando ai materiali reperibili in rete, sono i sintagmi fenomeno valanghivo, usato specialmente al plurale, (circa 3700 occorrenze al 26.02.2010), attività valanghiva 
(2900), evento valanghivo (I70o), il già citato rischio valanghivo usato quasi esclusivamente al singolare (I.280 occorrenze), sito valanghivo (circa 80o); più rari (alcune centinaia di occorrenze) pericolo valanghivo e incidente valanghivo e le associazioni situazione, area, dinamica, origine valanghiva, fino alle rarissime fase, fenomenologia e criticità valanghiva, con attestazioni per qualche unità. Se si esaminano questi sintagmi si vede che la relazione espressa dall'aggettivo è variabile: "incidente provocato, caratterizzato da valanga", "rischio, pericolo di valanghe", "siti, aree soggetti a valanghe", "situazione, dinamica, fenomeno, evento relativo a valanghe".

Per coniare un aggettivo capace di esprimere tutte questi relazioni si è aggiunto al sostantivo valanga il suffisso -ivo, usato in un primo tempo solo per la derivazione dalla forma del participio passato e successivamente esteso anche ai sostantivi per derivarne appunto aggettivi che indicano qualità, capacità, disposizione, relazione (abusivo, aggressivo, boschivo, detersivo, distintivo...). Nella formazione di valanghivo credo si possa a ragione ipotizzare l'influenza di eruttivo, termine introdotto in lingua nel XVIII secolo (DELI: 1765 ), riconducibile allo stesso ambito scientifico e che compare assai frequentemente associato ai sostantivi attivitá ed evento. D'altra parte non sono pochi gli aggettivi uscenti in -ivo usati in geografia, geologia o geofisica (intrusivo, estrusivo in riferimento alle rocce, corrosivo, erosivo riferiti all'azione degli agenti atmosferici, diffusivo, effusivo riferiti al magma...).

Per quel che riguarda l'esistenza di una possibile alternativa a valanghivo, nessuno dei dizionari consultati riporta un altro aggettivo derivato da valanga; è vero però che, sempre in ambiente tecnicoscientifico, circola da alcuni decenni il termine valangoso: si tratta di una derivazione prodotta tramite il suffisso -oso, già presente in aggettivi di origine latina, assai produttivo in italiano per formare "aggettivi denominali o deaggettivali che esprimono la presenza, la rilevanza, la qualità, l'effetto di ciò che è indicato dal sostantivo di base" (GRADIT). La forma è stata coniata molto probabilmente per analogia con franoso, molto usato nel sintagma terreno franoso, che riguarda un fenomeno dalla dinamica molto simile a quella della valanga. Le prime attestazioni di valangoso sembrano risalire alla fine degli anni Venti: si trova in un Trattato di patologia e terapia vegetale ad uso delle scuole di agricoltura di Teodoro Ferraris, edito nel I927, nella Rivista del Club Alpino Italiano, anno I928, vol. 47, e in Istituzioni di idronomia montana ad uso dei forestali e degli ingegneri, edito nel i93o. L'impiego del termine in testi tecnici (di agricoltura, geologia, geofisica, talassografia) continua fino agli anni '5o del secolo scorso, mentre successivamente sembra restringersi alla produzione degli "utenti" della montagna: lo si trova prevalentemente in siti curati da gestori di rifugi, da associazioni di trekking montano, dallo stesso Club Alpino Italiano; sembra non essere penetrato invece, a differenza di valanghivo, nel linguaggio delle amministrazioni o di enti a esse correlati. È da notare inoltre che, mentre valanghivo appare capace di coprire tutte le relazioni espresse da valangoso, non è possibile affermare il contrario: non sono ammissibili infatti i sintagmi rischio, pericolo o incidente valangoso; d'altra parte, anche nei contesti in cui è più frequentemente testimoniato - in associazione a canale o canalone, strato (di neve), ambiente, terreno - valangoso appare decisamente più raro rispetto a valanghivo.

\section{Cita come:}

Matilde Paoli, L'aggettivo valanghivo riferito a valanga, "Italiano digitale", 2010, XI, 2019/4 (ottobredicembre)

DOI: $10.35948 / 2532-9006 / 2020.3265$

Copyright 2010 Accademia della Crusca

Pubblicato con licenza creative commons CC BY-NC-ND 